\title{
CIENCIA\&SALUD
}

\section{Fisiopatología y presentación clínica del}

hiperaldosteronismo primario. Revisión de tema.

\section{Pathophysiology and clinical presentation of primary} hyperaldosteronism. Topic review.

Alexandre Monge Villalobos ${ }^{1}$ Ariana Echeverri Lohrengel ${ }^{2}$ Daniel Francisco Leiva Rojas ${ }^{3}$ Priscilla Soto Rivera ${ }^{4}$ María José Salas Fernandez ${ }^{5}$

1,2 ,3, 4 y 5 Médico general. Trabajador independiente, San José Costa Rica.

Contacto: alemonge0206@gmail.com

\section{RESUMEN}

El hiperaldosteronismo tiene una prevalencia cercana al 10-15\% entre la población de pacientes hipertensos y es comúnmente subdiagnosticada, especialmente en población joven. Nueva evidencia ha cambiado la manera en la que se diagnostica esta patología, cambiando la idea antigua de que el exceso de mineralocorticoides se presenta en su mayoría con hipertensión e hipokalemia; sin embargo, a la luz de nuevos estudios se acepta que solo la minoría de los pacientes se presenta con hipokalemia, haciendo de este, un hallazgo inconsistente.

Palabras Clave: aldosterona, hiperaldosteronismo, hiperaldosteronismo primario, fisiopatología del hi-peraldosteronismo.

\section{Cómo citar:}

Monge, A., Echeverri Lohrengel, A., Leiva Rojas, D. F., Soto Rivera, P., \& Salas Fernandez, M. J. . Fisiopatología y presentación clínica del hiperaldosteronismo primario.: Revisión de tema. Revista Ciencia Y Salud, 5(3), Pág. 35-41. https://doi. org/10.34192/cienciaysalud.v5i3.292

Recibido: 26/ene/2021 Aceptado: 09/may/202 Publicado:22/jun/2021

\section{ABSTRACT}

Hyperaldosteronism has a prevalence close to $10-15 \%$ among the population of hyper-tensive patients and is commonly underdiagnosed, especially in the young population. New evidence has changed the way in which this pathology is diagnosed, changing the old idea that mineralocorticoid excess presents mostly with hypertension and hypoka-lemia, however, in light of new studies, it is accepted that only the minority of patients present with hypokalemia, making this an inconsistent finding

Keywords: aldosterone, hyperaldosteronism, primary hyperaldosteronism, pathophysiology of hy-peraldosteronism.

\section{METODOS}

Para la elaboración de este artículo se realizó una investigación que incluyó artículos y publicaciones científicas con palabras como: aldosterona, hiperaldosteronismo, hipe-raldosteronismo primario, fisiopatología del hiperaldosteronismo. Se consultaron bases de datos como: UpToDate, Scielo, PubMed y The new England Journal of Medicine. Se consultó solo literatura en inglés 


\section{CIENCIA\&SALUD}

\section{ANTECEDENTES}

Prevalencia de hiperaldosteronismo primario - La literatura clásica sugiere que la prevalencia del hiperaldosteronismo primario es cercano a 1\% de los pacientes con hi-pertensión arterial, sin embargo, en estudios recientes se documenta una prevalencia considerablemente mayor (1).

Presentación variable - En la actualidad se cuenta con datos que indican que la ma-yoría de los pacientes con exceso de mineralocorticoides tiene concentraciones norma-les de potasio plasmático.

Según la nueva evidencia, la presencia de hipokalemia es hallazgo inconsistente (2). In-cluso en muchas ocasiones, la presencia de hipokalemia en pacientes hipertensos con hiperaldosteronismo primario que presentan hipokalemia se encuentra más ligada al uso de diuréticos no ahorradores de potasio que con la fisiopatología misma de la en-fermedad. Esto aleja aún más este hallazgo de los criterios diagnósticos del hiperaldos-teronismo primario.

Importancia - La identificación de esta patología tiene una gran importancia debido al aumento de morbilidad y mortalidad asociada a aumento del riesgo cardiovascular si se compara con población con las mismas características epidemiológicas y cifras simila-res de presión arterial.

\section{INTRODUCCIÓN}

La hipersecreción no suprimible de aldosterona es una causa subdiagnosticada de hi-pertensión arterial. El diagnóstico clásico se basa en una triada diagnóstica que consis-te en la presencia de hipertensión, alcalosis metabólica e hipokalemia, sin embargo, ac-tualmente se conoce la baja incidencia de hipokalemia en la presentación moderna de la patología (4).

En este artículo se aborda la fisiopatología de la hipersecreción no suprimible de aldos-terona y la importancia de conocer las nuevas evidencias disponibles acerca de la pre-sentación del hiperaldosteronismo primario. Además, se hará una revisión sobre dife-rentes tipos de hipersecreción de mineralocorticoides, causas, cuadro clínico, importan-cia clínica y diagnóstico temprano de esta patología.

\section{SUBTIPOS DE HIPERALDOSTERONISMO PRIMARIO}

En la literatura clásica se habla de que un 1\% de los casos de hipertensión arte-rial se pueden atribuir a hipersecreción de aldosterona, sin embargo, la literatura moder-na indica que la hipersecreción de aldosterona puede ser responsable de hasta 5 a 13 porciento de los casos. A pesar de que la hipersecreción de aldosterona renina-independiente es un diagnóstico con reconocimiento creciente, sigue siendo subdiagnosticado.

Existen varios subtipos de aldosteronismo primario. Los más frecuentes son (3):

-Hiperaldosteronismo bilateral idiopático, también Ilamado hiperplasia bilateral idiopá-tica (60 a $70 \%$ de los casos de hiperaldosteronismo primario)

-Adenoma unilateral productor de aldosterona (30 a 40\% de los casos de hiperaldoste-ronismo primario)

Existen otras formas menos comunes, entre ellas se encuentran:

- Hiperaldosteronismo familiar

-Hiperplasia adrenal unilateral

- Carcinoma adrenocortical productor de aldosterona

-Tumores ectópicos productores de aldosterona

Adenomas unilaterales productores de aldosterona presentan mayores niveles de se-creción de aldoste- 


\section{CIENCIA\&SALUD}

rona, lo que resulta en hipertensión arterial más severa e hipokalemia más profunda, cuando esta está presenta, además, tiene mayor prevalencia en pacientes más jóvenes.

Hiperaldosteronismo bilateral idiopático genera una forma más leve de la enfermedad.

\section{ALDOSTERONA}

La aldosterona es una hormona esteroidea de la familia de mineralocorticoides produci-da por la sección externa de la zona glomerular de la corteza de la glándula suprarrenal. Actúa en la homeostasis del sodio, potasio y en la regulación de la presión arterial. La angiotensina II es el estímulo más potente para la secreción de la aldosterona, mediante la acción sobre el receptor AT1R en la glándula suprarrenal.

Ejerce su mecanismo de acción mediante la expresión de canales epiteliales de sodio en el túbulo distal renal, que resulta en aumento de la reabsorción de agua y sodio que conlleva aumento de la excreción de potasio (3).

La aldosterona desarrolla un papel importante en la enfermedad renal y cardiovascular.

\section{FISIOPATOLOGÍA}

El hiperaldosteronismo primario es una patología caracterizada por la sobreproducción de aldosterona que resulta en un exceso de este mineralocorticoide en el plasma, cau-sando con frecuencia aumento de riesgo cardiovascular. Los efectos renales de la al-dosterona causan expansión de volumen, hipertensión arterial e hipokalemia. La secre-ción de aldosterona es relativamente independiente de la supresión del sistema renina-angiotensina-aldosterona.

Los signos clínicos presentes en el hiperaldosteronismo primario derivan principalmen-te de su efecto a nivel renal. El efecto primario de la aldosterona consiste en generar un incremento del número de canales de sodio en estado abierto presentes en la membrana luminal de las células principales en el túbulo colector cortical; esto aumenta la tasa de reabsorción de sodio (1).

Al incrementarse la tasa de reabsorción de sodio se genera una carga negativa en el lu-men del túbulo renal, esto facilita la excreción de potasio a través de los canales de po-tasio.

Al inicio, el exceso de mineralocorticoides genera retención de agua y sodio por el riñón, a esto le sigue un estado de diuresis espontánea llamado "escape de aldosterona". Este escape ocurre por sobrecarga de volumen que se genera por el aumento de la retención de agua, usualmente después de $3 \mathrm{~kg}$ de ganancia de volumen (1).

La sobrecarga de volumen generada por el exceso de mineralocorticoides produce que se activen sistemas de control por retroalimentación que inducen el llamado "escape de aldosterona", de este modo entran en acción moléculas como el péptido natriurético atrial (PNA), el cual ejerce su acción activando un mecanismo de diuresis por presión, el cual es estimulado principalmente por aumento de la presión arterial; esto hace que se disminuya la concentración de hormona antidiurética y con esto se logra aumentar la diuresis reduciendo la sobrecarga de volumen.

El mecanismo de control de hipokalemia por sí mismo contrarresta la excreción de pota-sio al llegar a niveles bajos de kalemia, lo cual genera una retención de potasio estimu-lado por hipokalemia. Cuando existe hipokalemia, a nivel del riñón se activa el cotrans-portador NKCC (cotransportador $\mathrm{Na}+/ \mathrm{K}+/ 2 \mathrm{Cl}$-) del túbulo contorneado distal, lo que au-menta la reabsorción de potasio, pero más importante aún, disminuye la carga de sodio que llega al túbulo distal, esto genera que se disminuya la tasa de reabsorción de sodio y que no se genere el gradiente electroquímico para la hipersecreción de potasio. Así, la hipokalemia misma activa un retrocontrol para disminuir la pérdida de potasio (5).

Otro de los mecanismos de control que impiden la excreción excesiva de potasio se ge-nera por el aumento de la actividad de la angiotensina II a nivel de la membrana luminal del túbulo colector cortical, la cual 


\section{CIENCIA\&SALUD}

reduce la actividad de los canales secretores de po-tasio (ROMK) (5).

Los efectos del exceso de aldosterona se equilibran con los reflejos de retroalimenta-ción que impiden alteración excesiva de la homeostasis. La pérdida de sodio y potasio cuando se alcanza este estado de equilibro es igual a la ingesta (6).

\section{MUTACIONES GENÉTICAS}

Mutaciones del gen KCNJ5 están presentes en $40 \%$ de los pacientes con APA. Este gen se ve implicado en el funcionamiento de un canal iónico para potasio en las células de la zona glomerulosa de la glándula suprarrenal. La activación de este gen produce despolarización de la célula, lo que permite la entrada de calcio a la célula, y este au-mento del nivel de calcio en la célula es la señal para la producción de aldosterona (15).

Un estudio multicéntrico arrojó resultados que indicaron que las mutaciones somáticas del gen KCNJ5 estuvieron presentes en 34\% (129 de 380) de los pacientes con APA, con una incidencia mucho mayor en el sexo femenino (14).

Mutaciones somáticas menos frecuentes en los genes ATP1A1, ATP2B3, CACNA1D y CTNNB1 se han visto asociadas a la aparición de hiperaldosteronismo primario (3).

\section{CARACTERÍSTICAS CLÍNICAS}

La hipersecreción de aldosterona no suprimible y relativamente independiente de renina es una causa subdiagnosticada de hipertensión refractaria a tratamiento que, si bien es cierto, su existencia cada vez es más conocida, aún sigue teniendo tasas bajas de diag-nóstico.

La presentación clásica del hiperaldosteronismo se basa en pacientes con hipertensión arterial refractaria a tratamiento óptimo ( 3 drogas incluido un diurético) que asocian hi-pokalemia y alcalosis metabólica.

La información actual indica que solo un 37\% de los pacientes con hiperaldosteronis-mo primario se presenta con hipokalemia (3).

En general, cuando se comparan pacientes con hiperplasia adrenal idiopática, los pa-cientes con adenomas productores de aldosterona tienden a tener niveles más altos de hipertensión arterial y de hipokalemia más profunda, cuando esta está presente (1).

Riesgo cardiovascular - El riesgo cardiovascular es independiente de los niveles plasmáticos de potasio y se asocia a hipertrofia del ventrículo izquierdo y disfunción del ventrículo izquierdo (7).

El exceso de aldosterona se asocia a un aumento del riesgo cardiovascular indepen-diente del nivel plasmático de potasio. La acción de la aldosterona sobre receptores mi-neralocorticoides produce inflamación, fibrosis y neovascularización en los vasos san-guíneos y el corazón, asociado a la reducción de la actividad de G6PD de estas células, impidiendo la adecuada función endotelial. Esto genera activación de cascadas de in-flamación, supresión de la acción del óxido nítrico e inclusive fibrosis cardíaca $(8,9)$.

Los niveles de hipertensión arterial en hiperaldosteronismo primario con frecuencia son marcadamente elevados. En un estudio se encontró que el promedio de presión arterial fue de 184/112 mmHg y 161/105 mmHg en pacientes con adenoma adrenal productor de aldosterona y en hiperplasia adrenal respectivamente (10). Sin embargo, a pesar de este hallazgo, la incidencia de hipertensión maligna es muy baja (11).

El aumento del riesgo cardiovascular puede revertirse según nuevos estudios randomi-zados, con el uso de antagonistas de receptores mineralocorticoides como espironolac-tona y eplerenona (12).

Efectos a nivel renal - La aldosterona parece aumentar la tasa de filtración glomerular y presión de perfusión renal por un mecanismo directo, independientemente de la pre-sión arterial sistémica. Además, se observa un incremento en la excreción de albúmina que puede indicar lesión renal (1). 


\section{CIENCIA\&SALUD}

Un artículo analizó a 50 pacientes con hiperaldosteronismo primario tratados de forma quirúrgica (adrenalectomía) o médica (espironolactona) contra 100 pacientes con hiper-tensión primaria que comenzaron tratamiento antihipertensivo. Al comienzo, en el esta-do basal de los pacientes, se identificó que la tasa de filtración glomerular y la tasa de secreción de albúmina eran mayores en los pacientes con hiperaldosteronismo primario. A los 6 meses de seguimiento, aún con control de presión arterial similar en ambos gru-pos, se determinó que la reducción en la tasa de filtración glomerular y la albuminuria fue significativamente mayor en el grupo de pacientes con hiperaldosteronismo (13).

\section{DIAGNÓSTICO}

El diagnóstico del hiperaldosteronismo primario es importante debido a su prevalencia, dentro de la población de pacientes hipertensos puede llegar a ser de un 10-15\%.

Existe un incremento del riesgo cardiovascular asociado a pacientes con hiperaldoste-ronismo primario, comparado con pacientes sin este padecimiento pero con los mismos niveles de presión arterial. Esto incrementa aún más la importancia del diagnóstico.

La meta de lograr un diagnóstico es tener la posibilidad de mejorar la hipertensión arte-rial y disminuir el riesgo cardiovascular asociado.

El diagnóstico tiene dos etapas que son el tamizaje y la confirmación.

\section{Tamizaje:}

Se realiza en pacientes con alta sospecha de hiperaldosteronismo primario, por ejemplo, pacientes con hipertensión refractaria a tratamiento óptimo.

Consiste en medir la actividad plasmática de la renina y la concentración plasmática de Aldosterona.

La evaluación inicial consiste en documentar que la actividad plasmática de renina y la concentración plasmática de renina estén disminuidas y la concentración plasmática de aldosterona esté aumentada.

\section{Confirmación del caso:}

Debe confirmarse al objetivizar la secreción inapropiada de aldosterona.

El examen debe realizarse en:

-Pacientes con hipertensión severa o resistente al tratamiento

-Hipertensión con incidentaloma adrenal

- Hipertensión en pacientes con historia familiar de hipertensión arterial, en familiares jóvenes o con historia de accidente cerebrovascular

-Pacientes con hipertensión y fibrilación atrial (18)

\section{CONFIRMACIÓN DEL DIAGNÓSTICO}

El examen de supresión de aldosterona usualmente es necesario para la confirmación del diagnóstico y puede realizarse tanto de manera oral como intravenosa (19).

Para realizar el examen vía oral, lo usual es administrar cargas orales altas de sodio du-rante tres días. Luego del tercer día, se recolecta una muestra de orina de 24 horas para la medición de sodio, aldosterona y creatinina. Una secreción urinaria de aldosterona mayor a 12 microgramos en 24 horas es un hallazgo consistente con hiperaldostero-nismo primario.

Para realizar el examen vía intravenosa, se administran 2 litros de solución salina isotó-nica en un periodo de 4 horas, idealmente con el paciente sentado. Una concentración plasmática de aldosterona sobre los 10 


\section{CIENCIA\&SALUD}

ng/dL es un hallazgo que también confirma el diagnóstico de hiperaldosteronismo primario.

Métodos para identificar los subtipos de hiperadosteronismo primario:

1. TAC con medio de contraste intravenoso: usualmente es el estudio inicial para diferenciar entre adenoma e hiperplasia y excluir el carcinoma adrenal.

2. Cateterización de venas adrenales: la medición de aldosterona en muestras de sangre venosa adrenal es el mejor criterio para distinguir entre hiperplasia bilate-ral y unilateral (20).

\section{CONCLUSIÓN}

El hiperaldosteronismo primario es una patología de la que cada día se conoce más y se diagnostica de forma más temprana; sin embargo, sigue siendo una entidad altamente subdiagnosticada. En la literatura clásica se habla sobre un patología que debe cumplir con dos criterios para su diagnóstico, hipertensión arterial más hipokalemia, sin embar-go, estudios actuales confirman que la hipokalemia es un hallazgo bastante inconsis-tente en la presentación de esta patología. La revisión de la fisiopatología del exceso primario de mineralocorticoides ayuda a entender por qué la hipokalemia es un hallazgo tan inconstante y nos da la oportunidad de tener un mayor conocimiento sobre el géne-sis de la patología para con el fin de brindar más herramientas para su diagnóstico tem-prano y con esto, disminuir el riesgo cardiovascular asociado a esta patología.

\section{REFERENCIAS BIBLIOGRÁFICAS}

3. Douma S, Petidis K, Doumas M, et al. Prevalence of primary hyperaldosteronism in resistant hypertension: a retrospective observational study. Lancet 2008; 371:1921.

4. Mattsson C, Young WF Jr. Primary aldosteronism: diagnostic and treatment strategies. Nat Clin Pract Nephrol 2006; 2:198.

5. Young WF Jr. Diagnosis and treatment of primary aldosteronism: practical clini-cal perspectives. J Intern Med 2019; 285:126.

6. Stewart PM. Mineralocorticoid hypertension. Lancet 1999; 353:1341.

7. Aron DC, Raff H, Findling JW. Effectiveness versus efficacy: the limited value in clinical practice of high dose dexamethasone suppression testing in the differen-tial diagnosis of adrenocorticotropin-dependent Cushing's syndrome. J Clin En-docrinol Metab 1997; 82:1780.

8. Xekouki P, Hatch MM, Lin L, et al. KCNJ5 mutations in the National Institutes of Health cohort of patients with primary hyperaldosteronism: an infrequent genetic cause of Conn's syndrome. Endocr Relat Cancer 2012; 19:255.

9. Monticone S, D'Ascenzo F, Moretti C, et al. Cardiovascular events and target or-gan damage in primary aldosteronism compared with essential hypertension: a systematic review and meta-analysis. Lancet Diabetes Endocrinol 2018; 6:41. 


\section{CIENCIA\&SSALUD}

10. Catena C, Colussi G, Nadalini E, et al. Cardiovascular outcomes in patients with primary aldosteronism after treatment. Arch Intern Med 2008; 168:80.

11. Monticone S, D'Ascenzo F, Moretti C, et al. Cardiovascular events and target or-gan damage in primary aldosteronism compared with essential hypertension: a systematic review and meta-analysis. Lancet Diabetes Endocrinol 2018; 6:41.

12. Blumenfeld JD, Sealey JE, Schlussel Y, et al. Diagnosis and treatment of primary hyperaldosteronism. Ann Intern Med 1994; 121:877.

13. Zarifis J, Lip GY, Leatherdale B, Beevers G. Malignant hypertension in associa-tion with primary aldosteronism. Blood Press 1996; 5:250.

14. Pitt B, Remme W, Zannad F, et al. Eplerenone, a selective aldosterone blocker, in patients with left ventricular dysfunction after myocardial infarction. N Engl J Med 2003; 348:1309.

15. Ribstein J, Du Cailar G, Fesler P, Mimran A. Relative glomerular hyperfiltration in primary aldosteronism. J Am Soc Nephrol 2005; 16:1320.

16. Boulkroun S, Beuschlein F, Rossi GP, et al. Prevalence, clinical, and molecular correlates of KCNJ5 mutations in primary aldosteronism. Hypertension 2012; 59:592.

17. Nanba K, Omata K, Else T, et al. Targeted Molecular Characterization of Aldoste-rone-Producing Adenomas in White Americans. J Clin Endocrinol Metab 2018; 103:3869.

18. Monticone S, D'Ascenzo F, Moretti C, et al. Cardiovascular events and target or-gan damage in primary aldosteronism compared with essential hypertension: a systematic review and meta-analysis. Lancet Diabetes Endocrinol 2018; 6:41.

19. Young WF. Primary aldosteronism: renaissance of a syndrome. Clin Endocrinol (Oxf) 2007; 66:607. 20. El Ghorayeb N, Mazzuco TL, Bourdeau I, et al. Basal and Post-ACTH Aldosterone and Its Ratios Are Useful During Adrenal Vein Sampling in Primary Aldosteron-ism. J Clin Endocrinol Metab 2016; 101:1826. 